\title{
Veranstaltungen
}

http://doi.org/10.1515/bd-2020-0025

\section{Fortbildungsprogramm 2020 Landesfachstelle für Öffentliche Bibliotheken in Thüringen}

\section{Organisatorische Hinweise \\ Teilnahme und Anmeldung}

Die Veranstaltungen richten sich an die Mitarbeiter/Innen der Öffentlichen Bibliotheken in Thüringen. Andere Interessierte können, wenn Plätze frei sind, zu den gleichen Bedingungen teilnehmen. Bitte melden Sie sich bis spätestens vier Wochen vor der Veranstaltung an. Ein Anmeldeformular finden Sie unter: https:// www.bibliotheken-thueringen.de/Fortbildungen.html.

Ihre Anmeldungen sind schriftlich, per Fax oder E-Mail möglich und werden in der Reihenfolge ihres Eingangs berücksichtigt. Sie sind verbindlich. Eine schriftliche Stornierung der Teilnahme wird bis zu zwei Wochen vor Beginn der Veranstaltung kostenfrei akzeptiert. Bei späteren Stornierungen sehen wir uns leider gezwungen, die Teilnahmegebühr in voller Höhe zu berechnen. Die anfallende Teilnahmegebühr ist bei der jeweiligen Veranstaltung aufgeführt. Hinzu kommen Ihre Dienstreisekosten. Sie erhalten von uns drei bis vier Wochen vor der jeweiligen Fortbildung eine gesonderte Einladung für Ihren Dienstreiseantrag. Unsere Fortbildungsveranstaltungen werden nur bei einer Beteiligung von mindestens zehn Teilnehmenden durchgeführt. Sollte eine Veranstaltung aufgrund zu geringer Anmeldungen nicht durchgeführt werden können, teilen wir das zwei Wochen vorher mit. Änderungen aus organisatorischen Gründen behalten wir uns vor. Bitte beachten Sie, dass Sie sich mit Speisen und Getränken selbst versorgen müssen.

Die Daten der Teilnehmenden (Name, Einrichtung, E-Mail-Adresse, Telefonnummer) werden ausschließlich zur Durchführung und Evaluation der Veranstaltung gespeichert und verarbeitet. Mit Ihrer Anmeldung erklären Sie sich mit der Speicherung und Verarbeitung einverstanden. Eine Weitergabe der Daten an unberechtigte Dritte erfolgt nicht. Sie können eine bereits erteilte Einwilligung jederzeit widerrufen. Dazu reicht eine formlose Mitteilung per E-Mail an die Landesfachstelle. 
Wenn Sie Anregungen für weitere Fortbildungsthemen haben, sind uns diese willkommen. Unser Fortbildungsangebot finden Sie auch im Internet unter: https://www.bibliotheken-thueringen.de.

\section{Anmeldungen richten Sie bitte an:}

Landesfachstelle für Öffentliche Bibliotheken in Thüringen

Heike Meier

Schillerstraße 40, 99096 Erfurt

Tel.: $0361262893-71$

Fax: $0361262893-79$

E-Mail: meier@bibliotheken-thueringen.de

\section{Fachtagung für Bibliotheksleiter/Innen in Öffentlichen Bibliotheken}

Themen: Neuerungen in der Deutschen Bibliotheksstatistik

Referentin: Gabriele Heugen-Ecker, Hochschulbibliothekszentrum des Landes Nordrhein-Westfalen

Leitung: Sabine Brunner, Landesfachstelle für Öffentliche Bibliotheken in Thüringen

Termin: 29. Januar 2020

Zeit: 9:30 bis 15:00 Uhr

Ort: Kinder- und Jugendbibliothek Erfurt, Marktstraße 21, 99084 Erfurt

Anmeldung: bis 8. Januar 2020

Die Deutsche Bibliotheksstatistik ist ein wichtiges Instrument, um die Leistungen der Bibliotheken sichtbar zu machen. Im Fragebogen zur DBS gibt es Änderungen, um ihn zu verschlanken und belastbare Daten $\mathrm{zu}$ erheben, die den neuen Entwicklungen in den Bibliotheken entsprechen, wie die Steuerungsgruppe zur DBS in ihrer Stellungnahme beschreibt. In der Veranstaltung werden die Änderungen vorgestellt und Fragen zur Deutschen Bibliotheksstatistik beantwortet.

Außerdem informiert die Landesfachstelle über neue Tendenzen und Entwicklungen im Bibliothekswesen sowie über aktuelle Projekte. Die Veranstaltung bietet die Gelegenheit, bibliotheksrelevante Fragen und Themen gemeinsam zu diskutieren. 


\section{Bücher ins Spiel bringen}

Referent: Thomas Wodzicki, Spiel- und Kulturpädagoge, Dozent für Erwachsenenbildung

Termin: 5. Februar 2020

Zeit: 9:30 bis 15:00 Uhr

Ort: Kinder- und Jugendbibliothek Erfurt, Marktstraße 21, 99084 Erfurt

Anmeldung: bis 8. Januar 2020

Teilnahmegebühr: 20,- Euro

„Alarm, Alarm ... - bei Oma Eierschecke ist der Kuchen angebrannt“ so kann man im Kinderbuch „Bei der Feuerwehr wird der Kaffee kalt“ lesen. Wer kennt dieses Buch nicht? Viele Kinder lieben es, sich zu verkleiden und in die Rollen der Erwachsenen bzw. ihrer Buchheldinnen und Buchhelden zu schlüpfen und Figuren zum Leben $\mathrm{zu}$ erwecken. In der Fortbildung wird es darum gehen, wie z. B. ein Besuch im Zoo oder die Geschichte der Arche Noah spielerisch nachempfunden werden kann als auch zu welchen Büchern wir den Kindern einen spielerischen Einstieg ermöglichen wollen. Zudem werden Inhalte der Fortbildung sein: Vermittlung von Buchinhalten über Spiele; Vorstellung von Lieblingsbüchern; Austausch zur aktuellen Nutzung von Kinderbüchern; spielpädagogische Arbeit mit Büchern; Einsatz von Musik, Verkleidung, Raumgestaltung und Tanz bei der spielerischen Umsetzung von Kinderbüchern.

Der Workshop teilt sich in zwei Abschnitte. Im ersten Teil werden Bilderbücher und Jugendbücher spielerisch vorgestellt. Im zweiten Teil werden gemeinsam Spiele, Aneignungs- und Präsentationsformen zu den Büchern entwickelt und erprobt. Bitte bringen Sie ein Buch aus ihrem Bestand mit, zu dem Sie gern einen spielerischen Zugang entwickeln möchten.

Aufgrund des Workshopcharakters der Fortbildung ist die Teilnehmendenzahl begrenzt.

\section{Begeistern mit Stimme, Sprache und Storytelling}

Referentin: Laura Cadio, Theaterpädagogin, Kulturvermittlerin

Termin: 18. März 2020

Zeit: 10:00 bis 15:30 Uhr

Ort: Kinder- und Jugendbibliothek Erfurt, Marktstraße 21, 99084 Erfurt Anmeldung: bis 19. Februar 2020

Teilnahmegebühr: 20,- Euro 
Wie wirke ich vor einer Gruppe? Ist meine Stimme laut und klar genug? Schaffe ich es durch meine Präsenz, die Aufmerksamkeit zu gewinnen? Wie kann ich meine Stimme, Mimik und Gestik und die Art wie ich etwas erzähle einsetzen, um andere zu begeistern? Im Workshop geht es darum, mit theatralen Methoden und spielerischer Herangehensweise Literatur zu vermitteln und Neugierde $\mathrm{zu}$ wecken. Methoden aus dem Theater werden genutzt, um die Stimme zu schulen und Geschichten zum Leben zu erwecken. Gemeinsam wird gespielt und ausprobiert, um so selber zu erfahren, wie es ist, die Geschichten lebendig werden zu lassen. Unterschiedliche Übungen und Methoden aus dem Theaterbereich werden selbst ausprobiert, um die eigene Arbeit zu ergänzen.

Aufgrund des Workshopcharakters der Fortbildung ist die Teilnehmendenzahl begrenzt.

\section{Bücher, über die man spricht}

Neuerscheinungen auf dem Buchmarkt

Referentin: Claudia Stapp, Buchhändlerin, Inhaberin „Buch Stapp“ Erfurt

Termin: 25. März 2020

Zeit: 9:30 bis 15:00 Uhr

Ort: Kinder- und Jugendbibliothek Erfurt, Marktstraße 21, 99084 Erfurt

Anmeldung: bis 26. Februar 2020

Claudia Stapp, Buchhändlerin und Inhaberin der Buchhandlung „Buch Stapp“ in Erfurt, präsentiert Ihnen nicht nur die Neuerscheinungen der Leipziger Buchmesse, sondern auch Novitäten, die im Sommer und Frühherbst 2020 erscheinen werden. Neben einer Reihe von neuen Romanen und Bestsellern werden Neuerscheinungen im Bereich der Kinder- und Jugendliteratur mit einem kurzen Abstecher zur Sachliteratur kurzweilig und informativ vorgestellt. Alle Themenbereiche werden mit ausreichenden Anschauungsmaterialien untersetzt. Darüber hinaus informiert Sie Claudia Stapp über Trends und Entwicklungen des gesamten Buchmarktes. 


\section{Der Einsatz von Tablets am Beispiel Thüringer Bibliotheken}

Leitung: Sabine Brunner, Landesfachstelle für Öffentliche Bibliotheken in Thüringen

Termin: 22. April 2020

Zeit: 9:30 bis 15:00 Uhr

Ort: Kinder- und Jugendbibliothek Erfurt, Marktstraße 21, 99084 Erfurt Anmeldung: bis 25. Marz 2020

Die Unterstützung der Entwicklung von Lese-, Medien- und Informationskompetenz ist eine wichtige Aufgabe für Öffentliche Bibliotheken. Dabei spielt der Einsatz von Tablets eine große Rolle. Bibliotheken setzen Tablets in unterschiedlichen Projekten und mit unterschiedlichen Zielgruppen ein. Der Einsatz reicht von Bibliothekseinführungen über Ratespiele bis zu Vorlesestunden mit augmented reality. In der Veranstaltung werden verschiedene erprobte Projekte von Mitarbeiterinnen und Mitarbeitern aus Thüringer Öffentlichen Bibliotheken vorgestellt.

Die Veranstaltung teilt sich in zwei Abschnitte: Im ersten Teil stellen Bibliotheken verschiedener Größe Aktionen und Programme aus der Praxis vor. Im zweiten Teil werden die vorgestellten Aktionen und Programme in Kleingruppen besprochen, Erfahrungen ausgetauscht und weitere Ideen für die Praxis entwickelt.

\section{6. länderübergreifende Fortbildung der Fach- stellen aus Hessen, Sachsen, Sachsen-Anhalt und Thüringen}

Bibliotheksarbeit mit Kindern und Jugendlichen

Thema: Sprachentwicklung bei Kindern und Jugendlichen

Termin: 12. bis 13. Mai 2020

Ort: Stadtbibliothek, Salzstraße 35, 06618 Naumburg (Saale)

Teilnehmerzahl: max. 10 Personen

Anmeldung: bis 14. April 2020

Teilnahmegebühr: 20,- Euro

Anknüpfend an den Erfolg der letzten Jahre werden Sie bei der länderübergreifenden Fortbildungsveranstaltung der Fachstellen aus Hessen, Sachsen, Sachsen- 
Anhalt und Thüringen auch in diesem Jahr über neue Projekte, Trends und Entwicklungen in der Kinder- und Jugendbibliotheksarbeit informiert.

Interessierte erhalten rechtzeitig ein detailliertes Tagungsprogramm.

\section{Treffen Allegro-OEB-Anwenderbibliotheken}

Referierende: Tanja Heitsch, Joachim Oeding, Büchereizentrale Niedersachsen

Datum: 30. September 2020

Ort: Kinder- und Jugendbibliothek Erfurt, Marktstraße 21, 99084 Erfurt

Zeit: 11:00 bis 15:00 Uhr

Anmeldung: bis 2. September 2020

Dieser Workshop für alle Allegro-OEB-Anwenderbibliotheken in Thüringen bietet ein optimales Forum für den Erfahrungsaustausch zwischen den Nutzerinnen und Nutzern zur Klärung von Fragen und für Informationen zu Produktneuerungen. Bitte teilen Sie uns mit, welche konkreten Themen und welche Probleme besprochen werden sollen. Ihre Fragen und Themenvorschläge werden an die Referierenden weitergeleitet, damit die Veranstaltung bestmöglich auf Ihre Bedürfnisse ausgerichtet werden kann.

Auch Bibliotheken, die dieses System nicht nutzen, können am Anwendertreffen teilnehmen. Bitte beachten Sie, dass in diesem Fall gegebenenfalls eine Teilnahmegebühr anfällt. Bitte fragen Sie bei uns nach.

\section{Digitale virtuelle Bibliothek „Thuebibnet“ für Fortgeschrittene}

Referentinnen: Sabine Brunner, Elisabeth Greil, Landesfachstelle für Öffentliche Bibliotheken in Thüringen

Termin: 7. Oktober 2020

Zeit: 9:30 bis 13:00 Uhr

Ort: Kinder- und Jugendbibliothek Erfurt, Marktstraße 21, 99084 Erfurt Anmeldung: 9. September 2020

Die virtuelle Bibliothek „Thuebibnet“ wird kontinuierlich weiterentwickelt. Es werden neue Funktionen, neue Oberflächen oder Sicherheitsstandards abgebildet. Die Fortbildung gibt den Mitarbeiterinnen und Mitarbeitern der Verbund- 
bibliotheken die Gelegenheit, Neuerungen und Entwicklungen der Onlinebibliothek kennenzulernen, in Erfahrungsaustausch mit anderen Bibliotheken des Verbundes zu treten sowie Fragen zu klären, die in den Bibliotheken und bei den Nutzerinnen und Nutzern aufkommen.

\section{Qualitätsmanagement - Fragen und Antworten}

Referent: Dr. Fabian Sieber, Auditor Qualitätsmanagement in Öffentlichen Bibliotheken in Thüringen

Termin: 25. November 2020

Zeit: 9:30 bis 15:00 Uhr

Ort: Kinder- und Jugendbibliothek Erfurt, Marktstraße 21, 99084 Erfurt

Anmeldung: bis 28. Oktober 2020

Teilnahmegebühr: 20,- Euro

Der Workshop richtet sich an Bibliotheken, die über eine Teilnahme an der Initiative zum Qualitätsmanagement in Öffentlichen Bibliotheken in Thüringen nachdenken. Im Verlauf des Workshops soll ein Erfahrungsaustausch stattfinden und es werden Fragen zum Zertifizierungsverfahren beantwortet: Welche Voraussetzungen müssen erfüllt sein, um sich erfolgreich zu beteiligen? Welche Schritte bis zum Audit sind zu berücksichtigen? Wie lässt sich das Verfahren der Bewertung der eigenen Arbeit effektiv gestalten? Welche Erfahrungen mit dem Qualitätsmanagement gibt es und wie können diese genutzt werden?

Aufgrund des Workshopcharakters der Fortbildung ist die Teilnehmendenzahl begrenzt.

\section{Fachtagung für Bibliotheksleiter/Innen in Öffent- lichen Bibliotheken}

Thema: Ehrenamt in der Bibliothek

Referentin: Brigitte Manke, Geschäftsführerin, Thüringer Ehrenamtsstiftung Leitung: Sabine Brunner, Landesfachstelle für Öffentliche Bibliotheken in Thüringen

Termin: 2. Dezember 2020

Zeit: 9:30 bis 15:00 Uhr

Ort: Kinder- und Jugendbibliothek Erfurt, Marktstraße 21, 99084 Erfurt Anmeldung: bis 11. November 2020 
Öffentliche Bibliotheken benötigen, um den gewachsenen Aufgaben gerecht zu werden, hauptamtlich tätiges und fachlich ausgebildetes Personal. Zur Unterstützung des vielfältigen Dienstleistungsangebotes sind in vielen Bibliotheken Bürgerinnen und Bürger der Kommunen ehrenamtlich engagiert. Zum Einsatz Ehrenamtlicher entstehen die verschiedensten Fragen: Welche Aufgaben können ehrenamtliche Mitarbeiterinnen und Mitarbeiter übernehmen? Was muss ich beim Einsatz Ehrenamtlicher beachten? Wie finde ich ehrenamtliche Mitarbeiterinnen und Mitarbeiter? Solche und ähnliche Fragen sollen beantwortet werden.

Außerdem informiert die Landesfachstelle über allgemeine Entwicklungen und neueste Trends im Bibliothekswesen. Die Veranstaltung bietet die Gelegenheit, bibliotheksrelevante Fragen und Themen gemeinsam zu diskutieren.

\section{Kreistreffen der Öffentlichen Bibliotheken der Landkreise}

In den vergangenen Jahren haben sich Fachtreffen der Bibliotheksmitarbeiterinnen und -mitarbeiter auf Kreisebene etabliert und wurden zu einer festen Größe in unserem Fortbildungsangebot. $\mathrm{Zu}$ diesen Treffen der Öffentlichen Bibliotheken werden alle haupt-, neben- und ehrenamtlich geleiteten Öffentlichen Bibliotheken, Bürgermeister/Innen sowie die Kulturämter der jeweiligen Landkreise eingeladen. Wir informieren Sie über die Trends der Bibliotheksarbeit, stellen unsere aktuellen Angebote für Sie vor, sprechen über die weitere oder zukünftige Zusammenarbeit mit unserer Einrichtung und gehen auf Ihre Fragen und Probleme ein. Nutzen Sie diese Möglichkeit für Diskussionen und neue Anregungen für die eigene Arbeit.

Möchten Sie 2020 ein Treffen mit Beteiligung der Landesfachstelle für Öffentliche Bibliotheken in Ihrem Landkreis durchführen, melden Sie sich bitte bei der Landesfachstelle.

\section{Landkreise:}

\section{Eichsfeld}

Termin: 1. April 2020

Ort: Stadtbibliothek Heiligenstadt, Petristraße 32, 37308 Heilbad Heiligenstadt Anmeldung: bis 4. März 2020 


\section{Altenburg}

Termin: 17. Juni 2020

Ort: Stadtbibliothek Altenburg, Lindenaustr. 14, 04600 Altenburg

Anmeldung: bis 20. Mai 2020

\section{Kyffhäuserkreis}

Termin: 24. Juni 2020

Ort: Stadtbibliothek „Rudolf Hagelstange“, Markt 1, 99734 Nordhausen

Anmeldung: bis 27. Mai 2020

\section{Wartburgkreis}

Termin: 16. September 2020

Ort: Gemeindebibliothek Seebach, Am Stein 3, 99846 Seebach

Anmeldung: bis 19. August 2020

\section{Sömmerda}

Termin: 14. Oktober 2020

Ort: Stadt- und Kreisbibliothek Sömmerda, Weißenseer Str. 15, 99610 Sömmerda Anmeldung: bis 16. September 2020

\section{Hildburghausen}

Termin: 11. November 2020

Ort: Stadt- und Kreisbibliothek „Joseph Meyer“, Markt 25, 98646 Hildburghausen Anmeldung: bis 14. Oktober 2020

\section{Gotha}

Termin: 18. November 2020

Ort: Stadtbibliothek Ohrdruf, Schlossplatz 1, 99885 Ohrdruf

Anmeldung: bis 21. Oktober 2020 


\section{Einladung: Fachtagung „Zukunft wissenschaftlicher Bibliotheken?!“}

ZB MED - Informationszentrum Lebenswissenschaften und das Fachmagazin Open Password veranstalten gemeinsam am Donnerstag, den 23. Januar 2020 am Kölner Standort von ZB MED die Fachtagung „Zukunft wissenschaftlicher Bibliotheken?!“. Eingeladen sind insbesondere Lehrende, Forschende und Studierende der Informationswissenschaft, der Lebenswissenschaften und angrenzender Disziplinen, aber auch Entscheider und Mitarbeitende wissenschaftlicher und öffentlicher Bibliotheken sowie engagierte Nutzende.

Die Veranstaltung geht der Frage nach, ob wissenschaftliche Bibliotheken eine Zukunft haben und gibt direkt die Antwort: In der Keynote erörtert Dr. Dietrich Nelle, ehemaliger Interimsdirektor von ZB MED, die Perspektiven wissenschaftlicher Bibliotheken und zeigt Lösungsmöglichkeiten für die anstehenden Herausforderungen auf.

In mehreren Impulsvorträgen geben Expertinnen und Experten aus unterschiedlichen Blickwinkeln Anregungen und stellen zukunftsweisende Projekte und Entwicklungen vor. Anne Christensen, die ab dem 1. Januar 2020 aus der Leuphana-Bibliothek als strategische Bibliotheksberaterin in die Wirtschaft wechselt, spricht über Strategien für digitale Dienste; ihre Thesen zur Zukunft wissenschaftlicher Bibliotheken bilden die Grundlage für den Beitrag. Prof. Dr. Dietrich Rebholz-Schuhmann, Wissenschaftlicher Direktor von ZB MED, zeigt auf, dass sich Forschung und Bibliothek innerhalb einer Einrichtung gegenseitig ergänzen und auf Augenhöhe zusammenarbeiten. Prof. Dr. Sören Auer, Direktor der TIB - Leibniz-Informationszentrum Technik und Naturwissenschaften, erläutert den Transformationsprozess wissenschaftlicher Bibliotheken von dokumentenbasierten $\mathrm{zu}$ wissensbasierten Informationsflüssen und schildert, welche Schritte seine Einrichtung bereits in diese Richtung unternommen hat. Prof. Dr. Konrad Förstner, bei ZB MED Leiter der Informationsdienste und zugleich Professor an der TH Köln, thematisiert die Qualifizierungen für zukünftige Anforderungen in Bibliotheken und stellt den neuen Zertifikatskurs zum „Data Librarian“ vor. Die abschließende Diskussion beleuchtet die Rolle der wissenschaftlichen Bibliotheken als unverzichtbarer Kooperationspartner der Wissenschaft.

Die Fachtagung schließt an das 2019 erschienene Buch „Zukunft der Informationswissenschaft - Hat die Informationswissenschaft eine Zukunft?“ an. Eine erste Veranstaltung am 5. September 2019 an der TU Berlin nahm die Zukunft der Informationswissenschaft in den Fokus. Da das Buch als zentrales Thema auch die Perspektiven wissenschaftlicher Bibliotheken beleuchtet, folgt nun auch zu diesem Schwerpunkt eine Veranstaltung. Bibliotheken stehen ebenso wie ihre 
Klientel an den Hochschulen und Forschungseinrichtungen vor großen Herausforderungen. Open Access, Forschungsdatenmanagement, Informations- und Datenkompetenz, Langzeitarchivierung, Künstliche Intelligenz, Citizen Science, Personalgewinnung als neuer Engpass, die Anpassung der bibliothekswissenschaftlichen Lehre an neue Anforderungen - um nur diese zu nennen. Einige wissenschaftliche Bibliotheken wie zum Beispiel ZB MED begegnen dem mit der Intensivierung ihrer Forschung und stehen dadurch in einem bedeutenden Strukturwandel. Dr. Willi Bredemeier, der das Buch zur Zukunft der Informationswissenschaft mit zahlreichen Fachbeiträgen renommierter Expertinnen und Experten herausgegeben hat, gibt bei der Fachtagung „Zukunft wissenschaftlicher Bibliotheken?!“ ein Statement zum aktuellen Stand der Debatte.

\section{Alle Infos auf einen Blick:}

- Was: Fachtagung „Zukunft wissenschaftlicher Bibliotheken?!“

- Wer: veranstaltet von ZB MED - Informationszentrum Lebenswissenschaften, dem Institut für Informationswissenschaft der TH Köln und Open Password

- Wann: Donnerstag, 23. Januar 2020, ab 14.00 Uhr

- Wo: ZB MED, Standort Köln, Gleueler Straße 60, 50931 Köln

\section{Programm:}

14.00 Uhr Registrierung - Meet and Greet

14.30 Uhr Willkommen!

Gabriele Herrmann-Krotz, Kaufmännisch-administrative Geschäftsführerin $Z B M E D$

14.35 Uhr Statement: Die Debatte geht weiter - zum Buch „Zukunft der Informationswissenschaft“

Dr. Willi Bredemeier, Herausgeber

14.45 Uhr Keynote: Herausforderungen, Lösungen und Perspektiven wissenschaftlicher Bibliotheken

Dr. Dietrich Nelle, $B M B F$, ehemaliger Interimsdirektor $Z B M E D$

15.15 Uhr Impulsreferat: Digitale Dienste in Bibliotheken - Thesen für erfolgreiche Strategien

Anne Christensen, derzeit Universitätsbibliothek Leuphana, ab 1. Januar 2020 strategische Bibliotheksberatung bei Effective Webwork

15.30 Uhr Impulsreferat: Das forschende Informationszentrum und seine lebenswissenschaftliche Bibliothek auf Augenhöhe Prof. Dr. Dietrich Rebholz-Schuhmann, ZB MED 
15.45 Uhr Impulsreferat: Von dokumentenbasierten zu wissensbasierten Informationsflüssen - Die wissenschaftlichen Bibliotheken im Transformationsprozess

Prof. Dr. Sören Auer, Technische Informationsbibliothek Hannover

16.00 Uhr Impulsreferat: Qualifizierung für die Anforderungen von morgen - Ausbildung zum Data Librarian

Prof. Dr. Konrad Förstner, ZB MED und Institut für Informationswissenschaft der TH Köln

16.15 Uhr Diskussion unter Einbeziehung des Plenums: Die wissenschaftlichen Bibliotheken als unverzichtbarer Kooperationspartner der Wissenschaft

Mit Sören Auer, Anne Christensen, Elisabeth Müller (ZB MED), Dietrich Nelle und Dietrich Rebholz-Schuhmann Moderation: Konrad Förstner

\subsection{Uhr Empfang}

Wir freuen uns, Sie in Köln zu begrüßen! Für unsere Planung möchten wir Sie bitten, sich bis zum 17. Januar 2020 zur Fachtagung anzumelden. Die Teilnahme ist kostenlos.

\section{Weitere Informationen:}

- Zum Programm: https://www.zbmed.de/fileadmin/user_upload/NewsBilder/Datei-Anhaenge/2020-01-23_Programm_Zukunft_wissenschaftlicher_ Bibliotheken.pdf

- Zur Anmeldung: http://survey.2ask.de/c9cf976c9c1dac3d/survey.html

Berichterstattung über die Veranstaltung zur Zukunft der Informationswissenschaft am 5. September 2019 in Open Password unter anderem am

- 23. September 2019: https://www.password-online.de/?wysija-page=1\&contr oller=email\&action=view\&email_id=791\&wysijap=subscriptions

- 30. September 2019: https://www.password-online.de/?wysija-page=1\&contr oller=email\&action=view\&email_id=793\&wysijap=subscriptions

- 21. Oktober 2019: https://www.password-online.de/?wysija-page=1\&controll er=email\&action=view\&email_id=799\&wysijap=subscriptions

„Zukunft der Informationswissenschaft - Hat die Informationswissenschaft eine Zukunft? - Grundlagen und Perspektiven - Angebote in der Lehre - An den Fronten der Informationswissenschaft“, herausgegeben von Willi Bredemeier - Simon Verlag für Bibliothekswissen, ISBN 978-3-945610-46-6, Berlin 2019. 